\title{
Current understanding of the role of microRNAs in spinocerebellar ataxias
}

\author{
Edyta Koscianska ${ }^{*}$ and Wlodzimierz J Krzyzosiak ${ }^{*}$
}

\begin{abstract}
The number of studies highlighting the role of microRNAs (miRNAs) in human physiology and diseases is growing, but many miRNA-driven regulatory mechanisms remain elusive. A proper understanding of the exact functions of individual miRNAs and their interaction with specific targets is vitally important because such knowledge might help cure diseases for which no effective treatment currently exists. Herein, we present current views on the role of the miRNA-mediated regulation of gene expression in the case of select spinocerebellar ataxias (SCAs) and their potential involvement in the pathogenesis of these diseases. Specifically, we summarize published data showing the known links between miRNAs and CAG repeat-dependent SCAs. Moreover, using the example of SCA type 3 (SCA3), we refer to the issue of prediction and validation of miRNA targets, and we demonstrate that miR-181a-1 may regulate the $3^{\prime}-U T R$ of the ATXN3 gene.
\end{abstract}

Keywords: Ataxin, PolyQ expansion, TREDs, miRNA, Target validation, Luciferase assay

\section{Introduction}

Several spinocerebellar ataxias (SCAs) belong to trinucleotide repeat expansion disorders (TREDs), which are a relatively large group of incurable dominantly inherited neurological diseases. More specifically, six SCAs belong to polyglutamine (PolyQ) diseases and are triggered by an expansion of the glutamine-coding CAG repeat, which is responsible for polyQ tract formation. A long polyQ stretch can elicit numerous aberrant cellular processes that lead to the degeneration and death of neuronal cells.

MicroRNAs (miRNAs) are potent regulators of gene expression. They act at the post-transcriptional level by inhibiting protein synthesis that occurs with or without transcript degradation. It appears that these small RNAs are engaged in a wide range of physiological and pathological activities in cells; therefore, their function and contribution to the pathogenesis of SCAs is of special consideration.

\section{Review}

This review summarizes the current knowledge regarding the putative involvement of miRNAs in the pathogenesis of CAG repeat-dependent SCAs and the experimentally

\footnotetext{
* Correspondence: edytak@ibch.poznan.pl; wlodkrzy@ibch.poznan.pl Department of Molecular Biomedicine, Institute of Bioorganic Chemistry, Polish Academy of Sciences, Noskowskiego 12/14 Str., 61-704 Poznan, Poland
}

proven associations between specific miRNAs and these diseases that have been reported. Moreover, it addresses some aspects of miRNA target prediction and validation using the example of our own research on the regulation of the ataxin-3 transcript.

\section{Brief characteristics of CAG repeat-dependent SCAs and miRNA-mediated regulation of gene expression}

There are six SCAs (SCA-1, 2, 3, 6, 7, and 17) among the polyQ diseases [1-3], and their common denominator is the expression of CAG repeats of abnormal length in the open reading frame (ORF) of the ataxin $(A T X N)$ genes. The pathogenic glutamine repeat threshold in most of these SCAs is approximately 35, except for SCA3 and SCA6, in which cases the pathogenic repeat range starts from 50 and 21, respectively. The ataxias that fall under the category of polyglutamine diseases have similar clinical presentations; however, molecular studies are necessary to distinguish particular SCA types. Current views on the identified molecular processes causing or modulating the neurodegenerative phenotype in spinocerebellar ataxias have been discussed recently and reviewed thoroughly [4].

The normal function of the polyglutamine proteins implicated in SCAs seems to be quite disparate and may be succinctly defined as follows: ataxin- 1 and ataxin-7 play a role in transcription regulation [5-7], ataxin-2 is involved 
in RNA metabolism and endocytosis processes [8,9], ataxin-3 interacts with transcriptional components and is responsible for de-ubiquitination and transcription regulation [10], ataxin-6 is linked to $\mathrm{Ca}^{+}$signaling/homeostasis [11], and ataxin-17 is linked to general transcription (TFIID complex) [12] (reviewed in [4]).

The expanded CAGs may trigger pathogenic effects based on three types of mechanisms: (1) toxic RNA gainof-function, (2) toxic protein gain-of-function, or (3) both transcript and protein loss-of-function $[3,13,14]$. The toxic protein gain-of-function mechanism explains neurodegeneration as the result of the expression of glutamine-rich proteins that can misfold and form aggregates, typically in nuclear inclusions, and eventually result in neuronal dysfunction and loss [15-17]. More recently, however, mutant CAG repeat transcripts have also been shown to contribute to the pathogenesis of polyQ diseases. Specifically, aberrant alternative splicing, transcript nuclear transport and export, RNA interference, and nucleolar stress resulting in apoptosis have been reported to be involved in CAG repeat RNA toxicity (reviewed in $[18,19]$ ).

To date, more than 2,500 mature human miRNAs have been deposited in the miRNA registry (miRBase, Release 20) [20]. These important molecules are endogenous short RNAs that downregulate gene expression by imperfect pairing with complementary sites within transcript sequences; miRNAs suppress their targets' translation, stimulate their deadenylation and degradation, or induce target cleavage (reviewed in [21]). Regarding the canonical biogenesis process, human miRNAs are generated by two RNase III endonucleases (Drosha and Dicer) that act sequentially in the nucleus and the cytoplasm (reviewed in [22-25]). The ribonuclease Drosha, acting together with the DGCR8 protein within the complex named Microprocessor, cleaves primary miRNA transcripts (pri-miRNA) into pre-miRNA precursors that are approximately 60 nucleotides long, while the ribonuclease Dicer processes pre-miRNAs into mature miRNA duplexes that are approximately 22 nucleotides long. Both canonical and non-canonical miRNA biogenesis pathways, the assembly of the miRNA-induced silencing complex (miRISC), and various intricate miRNA-mediated mechanisms of gene expression regulation have been recently discussed in detail [26].

Generally, the level of the polyQ-expanded protein is among the factors that contribute to disease severity [27-30]; however, the proposed cellular mechanisms that regulate protein levels remain to be fully characterized. miRNAs that are the key regulators of protein dosage in cells must therefore be directly linked with the pathogenesis of SCAs. The roles of miRNAs in the development and regulation of the nervous system and in a wide array of disorders of the nervous system have been proven in many studies (reviewed in [31-41]). Several miRNAs have been shown to target different neurodegenerative diseaserelated proteins and modulate their concentration in cells (e.g., BACE1, ataxin-1 or $\alpha$-synuclein in the case of Alzheimer's disease (AD), SCA1 and Parkinson's disease (PD), respectively $([42,43]$ and references therein)). Moreover, it was demonstrated that the dysfunction of the brainenriched miRNAs that specifically target and regulate the expression of disease-associated genes may lead to neurodegeneration and that disease-related proteins interact with the miRNA machinery [44-46]. Therefore, it is of growing importance to address the plausible involvement of miRNAs in the pathogenesis of particular SCA types and to identify the activities of individual miRNAs on SCA disease-coding genes.

\section{Links between miRNAs and CAG repeat-dependent SCAs}

Specific links between miRNA regulation and CAG repeatdependent SCAs have been described in several studies (reviewed in [47]) (Table 1). However, most of hitherto published reports focus on the role of miRNAs in the most common SCAs, namely SCA1 and SCA3.

\section{miRNAs in SCA1}

A study on SCA1 was the first to reveal that some miRNAs can regulate the expression of target transcript mRNA containing a CAG repeat expansion. More specifically, that study showed that miR-19a, miR-101, and miR-130a coregulate the 3'-UTR of ATXN1 through the inhibition of ATXN1 translation [48]. The gene coding for human ATXN1 has a long 3'-UTR region (approximately $7 \mathrm{~kb}$ ), which implies an important role in the post-transcriptional regulation governed by miRNAs. Moreover, cerebellar degeneration and ataxia in mice was reported to be interconnected with the impairment of miRNA biogenesis in Purkinje cells [49]. In a reporter assay, which is the simplest and most straightforward method of validation of miRNA-mRNA interactions, miRs 19a, 101, and 130a were shown to downregulate the expression of ATXN1 [48]. Interestingly, miR-101 affected both the mRNA and protein levels, whereas miR-19a and miR-130a decreased the protein levels only. Although these miRNAs exerted their effect in different cell lines when transfected individually, a markedly stronger effect was observed when miRs 19a, 101 and 130a were transfected together and cooperated in the regulation of their target. Moreover, inhibiting the endogenous expression of all three miRNAs in HEK293T cells with specific inhibitors and the mutagenesis of miRNA target sites in the 3 '-UTR of a hATXN1[86Q] expression vector enhanced the cytotoxicity of the mutant ATXN1 protein and significantly reduced cell viability. Taken together, these findings show that miRNAmediated posttranscriptional regulation of the ATXN1 gene may modulate SCA1-related neuropathology by affecting protein levels. 
Table 1 Reported changes in miRNA expression in SCAs and their links with polyQ toxicity

\begin{tabular}{|c|c|}
\hline Observed changes & miRNA prediction \\
\hline \multicolumn{2}{|l|}{ SCA1 } \\
\hline \multirow[t]{2}{*}{$\begin{array}{l}\text { miR-19, }-101 \text { and }-130 \\
\text { downregulate the ATXN1 gene }\end{array}$} & $\begin{array}{l}\text { Candidate miRNAs were } \\
\text { identified using the PicTar } \\
\text { algorithm. }\end{array}$ \\
\hline & $\begin{array}{l}\text { Eight miRNAs were chosen fo } \\
\text { further examination based on } \\
\text { the number of target sites in } \\
\text { the ATXN1 transcript and thei } \\
\text { neuronal expression. }\end{array}$ \\
\hline
\end{tabular}

miRNA expression upregulated in SCA1 patients; the increase more prominent in the cortex samples

miR-144 slightly downregulated in SCA1 cerebellum but strongly induced in the cortex Upregulated miRNAs predicted to target ATXN1, e.g., miR-101,

miR-144 and -101 downregulate ATXN1 expression, miR-25 does not affect ATXN1 levels $-130 a,-19 a,-302$

Regulatory RNA network and TargetScan prediction algorithms.

No evidence for a statistically significant difference in miRNA expression. A trend to overexpression of miR-33-5p, $-34-5 p$ and $-92 a-5 p$

34 miRNAs upregulated; 14 at both time points, 15 at the 4week time point only and 5 at the 12-week time point

12 miRNAs downregulated; 1 miRNA at both time points, 4 and 7 , respectively at the 4 and 12-week time points only

miR-150 levels increased in cerebellar Purkinje neurons and slightly decreased in granule cells

A concomitant increase in miR150 and decrease in Rgs8 and Vegfa levels

TargetScan; Rgs8 and Vegfa
Experimental methods

Transfection with miRNA duplexes and their specific $2^{\prime}$ O-methyl inhibitors followed by western blot and RT-PCR analyses. miRNAs transfected to cells either individually or collectively.

Luciferase reporter assays (Promega) with vectors carrying fragments or full length human ATXN1 3'-UTRs.

miRNA levels and their expression patterns in mouse cerebella were assessed by northern blot analysis and in situ hybridization with LNA probes (Exiqon)

Cell death assays with mutant

miRCURY LNA human microRNA Array (Exiqon).

qRT-PCR, TaqMan miRNA assays (Applied Biosystems) for miR144 and miR-101.

ATXN1 mRNA and protein levels analyzed with RT-PCR and western blot.

Overexpression of miRNA duplexes and miRNA inhibitors followed by western blotting.

Luciferase assays (Promega) with full-length ATXN1-3'-UTRhLuc reporters and miRNA duplexes or 2'-O-methyl modified masking oligos. miRs 144 and 101 tested separately and in combination.

Illumina Hi-Seq 2000, small RNAs from fly heads

miRCURY LNA all species microRNA arrays (Exiqon)

Individual miRNAs analyzed by qRT-PCR with TaqMan assays (miRs 150, 335, 23a, 24 and 143)

In situ hybridization using dig-labeled DNA-LNA probes (Exiqon) identified as targets of miR-150

Quantitative PCR and immunohistochemical analyses ATXN1deprived of target sites.

MCF7 (highly express endogenous ATXN1), HEK293T, HeLa and NIH3T3 cell lines

HeLa cell line

C57/B6 WT mouse

HEK293T cell line

Human cerebellum and cortex, SCA1 patients and healthy controls

HEK293T cell line

Drosophila models: UAS-Atxn182Q and UAS-Atxn1-30Q

Cerebellar RNA from SCA1 BO5 transgenic mice [82Q] analyzed at two time points (at 4 and 12 weeks of age)
SCA1 BO5 transgenic mice -

Purkinje neurons, granule cells

SCA1 cerebella, Purkinje neurons 


\section{Table 1 Reported changes in miRNA expression in SCAs and their links with polyQ toxicity (Continued)}

\author{
A dose-dependent decrease in \\ Vegfa expression induced by \\ the increased miR-150 activity
}

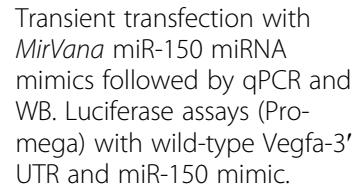

Transient transfection with MirVana miR-150 miRNA mimics followed by $\mathrm{QPCR}$ and WB. Luciferase assays (Promega) with wild-type Vegfa-3' UTR and miR-150 mimic.

\section{SCA2}

Upregulation of bantam and miR-12
Drosophila, transgenic

recombinant flies, wing

imaginal discs cells

\section{SCA3}

bantam miRNA is a

downstream modulator/

suppressor of polyQ toxicity

No evidence for a statistically significant difference in miRNA expression. A trend to a decrease in miR-1-3p and an increase in miR-100-5p, -33-5p and $92 a-5 p$ levels.

miR-34b is upregulated, and miR-25, -125b, -29a are downregulated in SCA3 patients. Expression of miR-25 and $-125 b$ was associated with the course of disease

\section{Phenotype mutants}

comparison analysis, TUNEL assays, immunostaining and western blots

Illumina Hi-Seq 2000, small RNAs from fly heads
Drosophila dcr-1 mutants, fly eyes, HeLa cells with normal and pathogenic Ataxin-3 treated with siRNA targeting dicer mRNA

Drosophila models: UAS-Atxn3$70 Q$ and UAS-Atxn3-19Q
[60]

57]

\section{SCA7}

No evidence for a statistically significant difference in miRNA expression. A trend to an increase in miR-33-5p and -92a$5 p$ levels and a decrease in miR-375-3p

Another key example of the potential involvement of miRNAs, especially miR-144, in brain aging and SCA1 pathogenesis has been provided by a genome-wide microarray analysis [50]. This study revealed a global age-related miRNA deregulation in the cortex and cerebellum of humans, chimpanzees and rhesus macaques. Interestingly, the vast majority of miRNAs in the examined samples were downregulated, and only a small fraction of miRNAs were found to be upregulated. A highly conserved miR-144 was found to be related to the aging process but regulated differently between species. Its expression was decreased in the human cortex but was consistently induced in the aging cerebellum and cortex of nonhuman primates. Moreover, a subsequent analysis of human samples showed a widespread activation of miRNA expression in the cortex and cerebellum of SCA1 patients relative to the miRNA expression in the brains of healthy individuals. The level of miR-144 that was predicted to bind to the ATXN1 3'-UTR was elevated in the cortex. Other miRNAs (e.g., miR-101, -130a, -19a, -302) predicted to target ATXN1 for degradation were also found to be upregulated. Next, the potential regulation of the ATXN1 transcript by the candidate miRNAs was examined using miRNA overexpression and inhibition as well as reporter assays. Of the three miRNAs tested, miRs 144 and 101 considerably decreased the endogenous ATXN1 protein levels (miR-25 activity was not observed). Similarly, these two miRNAs reduced reporter gene expression when tested either separately or collectively. Overall, it was concluded that the activation of specific miRNAs could serve to reduce the cytotoxic effect of the expanded mutATXN1 and that miRNA deregulation may be a risk factor for disease development. Protective functions for miRNAs have been also proposed by others $[33,37,51,52]$.

Global profiling of miRNA expression in a mouse model of SCA1 was performed to study alterations in miRNA expression in the cerebellum at both pre-symptomatic and symptomatic stages of pathogenesis [53]. More specifically, the analysis revealed changes in the levels of $46 \mathrm{miR}$ NAs in BO5 SCA1 transgenic mice [82Q], and a number of miRNAs displayed altered expression patterns before the onset of clear phenotypes. Mice were analyzed at two time points, i.e., 4 and 12 weeks. Of the miRNAs that were 
changed in the SCA1 mouse cerebella, 34 displayed increased expression; 14 of these miRNAs exhibited increased expression for both time points analyzed, and 15 and 5 exhibited increased expression at only the 4- or 12week time points, respectively. In contrast, the miRNA expression level was decreased for 12 miRNAs, one at both time points and 4 and 7 at the 4 - and 12-week time points, respectively. Importantly, the observed changes in miRNA expression (e.g., miR-150 and miR-335) were found immediately after the start of mutant Atxn1 expression in SCA1 mice and prior to phenotypic onset, showing that the deregulation of miRNA expression in the cerebellum of transgenic mice contributes to the early pathogenesis of SCA1. Moreover, in line with the fact that the expression of the mutAtxn1 protein is restricted to cerebellar Purkinje cells in SCA1 transgenic mice, elevated miR-150 expression was observed selectively in Purkinje neurons. In addition, the levels of two transcripts that are predicted targets of miR-150, Rgs8 and Vegfa, were reduced. The latter target was further validated in Neuro2A cells using miR-150 mimics and reporter assays; analyses of both types confirmed the functionality of binding sites for miR-150 that are present in Vegfa mRNA. Collectively, these data demonstrated that the expression levels of mutAtxn 1 and miR150 together with its target mRNA are highly interconnected. It was proposed that the mutAtxn1 de-represses activity at miRNA promoters, leading to an increase in miRNA levels, or acts through yet unknown a gain-oftoxic function mechanism. Therefore, evidence that miRNA misregulation during the critical post-natal period may underlay SCA1 pathogenicity was provided. Moreover, in this work, a promising therapeutic approach based on the RNAi-mediated suppression of mutant Atxn1 was presented. This therapeutic intervention for SCA1 has been developed further by the same group and described elsewhere [54].

The miRNA deregulation at the beginning of the pathological process has been also investigated in Drosophila transgenic model of SCA1 with the use of deep sequencing [55]. Specifically, two RNA samples from flies and one control were analyzed. The expression of miRNAs was evaluated at an early stage of the pathological process, 3 days after the induction of toxic proteins. Many of the selected miRNAs displayed low expression or highly variable expression between the tested samples. Although a trend toward the overexpression of miR-33, miR-34 and miR-92a was observed, no evidence for a statistically significant difference in miRNA expression at this early stage of the disease was found. Therefore, these results suggest that the toxicity in SCA1 in the early stages of the pathological process and the deregulation of miRNA expression most likely are not related.

\section{miRNAs in SCA3}

The miRNA bantam (ban) was identified as a downstream modulator/suppressor of polyQ toxicity in a Drosophila model of SCA3 that prevents degeneration [56]. Upregulation of the ban miRNA suppressed degeneration and diminished the toxicity of ataxin-3, which is the disease-causing protein for SCA3. Suppressing ban activity was connected with neither a change in the level of the pathogenic protein nor its accumulation and was shown to occur downstream of the polyQ protein accumulation. Moreover, reduced miRNA processing due to the knockout of Dicer1 enhanced the neurodegeneration induced by ataxin-3 in both flies and human cells by modulating pathways that normally contribute to polyQ toxicity [56]. The protective activity due to miRNAs was found to be specific because siRNA-dependent pathways did not modulate polyQ toxicity. Therefore, this research adds another piece of corroborating evidence that miRNA pathways dramatically modulate polyQinduced neurodegeneration.

A protective role of miRNAs in age-associated processes and polyQ disorders was further supported by demonstrating that miR-34b mitigates the toxicity of ataxin-3 in Drosophila [57]. Specifically, the upregulation of miR-34 in this fly model extended the median lifespan and reduced the neurodegeneration induced by human polyQ disease protein.

Drosophila transgenic model of SCA3 has been also used to investigate deregulation of miRNA expression by deep sequencing, as described for SCA1 with regard to the same analysis [55]. The study conducted at the beginning of the pathological process revealed a trend toward a decrease in miR-1 and an increase in miR-33, miR-92a and miR-100 levels, however, the observed changes in miRNA expression were not statistically significant.

Most recently, it has been demonstrated that miRNAs are useful biomarkers for SCA3, as specific changes in regulatory miRNA levels were identified in the serum obtained from patients suffering from this disease [58]. The microarray analysis validated by qRT-PCR revealed that miR-25, miR-125b, miR-29a and miR-34b were differentially expressed in SCA3 patients. Additionally, miR-29a and miR-34b showed the most dramatic changes in their expression levels, whereas the expression of miR-25 and mir-125b was associated with the course of disease. The latter miRNAs, miRs 25 and $125 \mathrm{~b}$, were found to bind to the 3 '-UTR of the ATXN3 gene. All miRNAs proposed by Shi et al. as potential biomarkers were earlier reported to be connected with neuronal physiology and pathology in terms of neurodegeneration [59-63]. Moreover, mir-34b was previously shown to be elevated in the plasma of premanifest Huntington's disease (HD) patients and predicted to be a protective factor for HD [60]. These findings, which 
advocate the use of serum miRNAs as biomarkers, are of potential value in the diagnosis of patients with neurodegenerative disorders.

miRNAs in other CAG repeat-dependent SCAs

The involvement of miRNAs in the pathogenesis of SCA7 has been investigated in Drosophila transgenic model of this disease, along with studying their role in SCA1 and SCA3 [55]. As aforementioned, global profiling of miRNA expression did not show significant changes in miRNA levels at an early stage of the pathological process in the examined SCAs. In the case of SCA7 the level of miR-33 and miR-92 tended to be higher and the level of miR-375 was found to be lower but these differences were not statistically significant. Therefore, no important associations between the toxicity induced by the expansion of CAG repeats in SCA7 and the deregulation of miRNA expression have been reported.

With regard to other SCA types, the link between neurodegeneration and miRNAs was demonstrated by showing that the gene encoding TATA-binding protein (TBP), which is mutated in SCA type 17 (SCA17), is downregulated by miR-146a [64]. Finally, some pathogenic proteins, such as ataxin-2, might be required for miRNA functioning; it was shown that the ataxin-2 protein, which interacts with Ago1, impairs the repressive activity of several miRNAs [65].

\section{Summary of miRNA-mediated gene regulation in SCAs}

All of the above-mentioned findings suggest that miRNA pathways can modulate polyQ-induced neurodegeneration but that the pathogenesis of polyQ diseases can also influence miRNA expression. It has been proven that miRNAs are implicated in the pathogenicity associated with SCAs at many levels of different pathological processes; however, miRNA specific functions remain largely unknown. Moreover, the question persists, how exactly miRNAs affect CAG repeat-mediated neurodegeneration and whether such miRNA activity is similar in all SCA types. A concept relating miRNAs and ataxias could be built on the known miRNA mode of action (Figure 1). The miRNA-mediated regulation of gene expression may be exerted by different mechanisms (reviewed in [26]), eventually leading to the reduction in levels of both factors responsible for the pathogenesis of polyQ diseases, i.e., mutant proteins and toxic transcripts [66]. The 3'-UTR regions of CAG repeat-dependent SCAs vary drastically in length, which may be important with regard to the fact that miRNA binding sites are mostly located in 3 '-UTRs of target genes. Although 3'-UTR lengths did not always correlate with the number of miRNA binding sites, the ATXN1 3'-UTR being the best-studied in terms of miRNA-mediated regulation is the longest and contains the greatest number of predicted miRNA binding sites [67]. Apart from direct regulation of genes triggering particular SCAs, miRNA may be also engaged in the

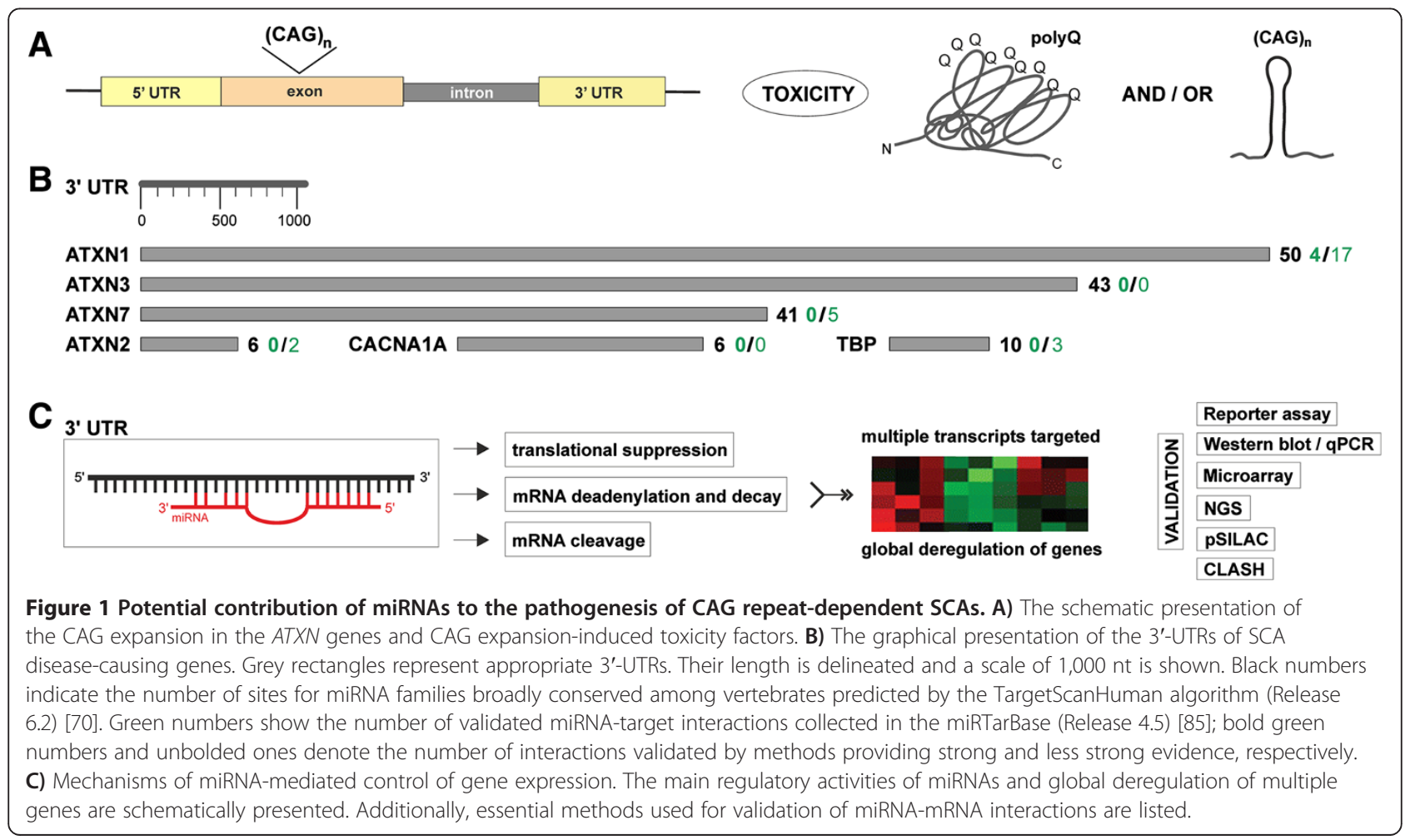


pathogenesis of these diseases indirectly by targeting multiple transcripts and affecting expression of numerous proteins. All in all, miRNAs regulatory potential contributes to the pathogenesis of CAG repeat-dependent SCAs.

\section{Prediction and validation of miRNA targets in SCA3}

Identifying biologically relevant miRNA targets is of paramount importance and involves the reliable prediction of miRNA-mRNA interactions and the positive validation of functionality of these interactions. Many different algorithms have been developed to predict miRNA-mRNA binding; computational tools such as miRANDA [68], PicTar [69], TargetScan [70], RNAhybrid [71] or PITA [72] have been successfully used for more than a decade, while the more recently invented programs miRco [73] and MREdictor [74] are currently coming into use. Similarly, various approaches for the experimental verification of predicted miRNA-mRNA interactions have been reported to date (reviewed in [75-77]). The most straightforward method for the verification of miRNA function is a transfection of cells with miRNA mimics or miRNA inhibitors, followed by quantitative analyses of target mRNA and protein levels $[78,79]$. There are also various high-throughput methods, such as the increasingly popular small RNA deep sequencing and microarrays as well as proteome and transcriptome analyses, which allow the identification of thousands of miRNA-target pairs $[80,81]$. Moreover, the most advanced and modern methods are based on UV crosslinking and immunoprecipitation followed by deep sequencing and bioinformatic mapping of the reads (reviewed in [82]). The simplest and most reliable method, however, is the use of luciferase reporter assays because miRNA activity on reporter genes can be easily measured $[69,83]$. Reporter systems enable the study of single specific miRNAmRNA interactions (reviewed in [84]) and have proven to be convenient and reliable in many studies (data and references collected in [85]). Therefore, they serve as an efficient and routinely used strategy for the verification of individual miRNA-mRNA interactions. More than 4,000 miRNA-target interactions have been validated by reporter assays, the collection of which is considered to provide strong experimental evidence accumulated in the miRTarBase $[85,86]$, which is the database containing the largest amount of such validated interactions $(51,460$ in total).

SCA3 (also known as Machado-Joseph disease) is among the most common dominantly inherited ataxias [87-89]. The post-transcriptional miRNA-mediated regulation of the disease-causing ATXN3 expression has been described only in few reports $[55,56,58]$ (Table 1 ). In our previous study, we performed an in-depth computational analysis of the miRNA interactions with all mRNAs derived from genes triggering hereditary neurological disorders known as trinucleotide repeat expansion diseases (TREDs) and showed that the gene mutated in the case of SCA3 may be subject to miRNA regulation [67]. From predicted potential interactions, we selected two for further experimental verification using a reporter system. Specifically, we have preliminarily validated a putative regulation of the ataxin-3 transcript by two miRNAs, miR-9-2 and miR-181a-1, by employing a luciferase assay and a set of reporter constructs as described previously [90,91]. Briefly, synthetic oligonucleotides corresponding to sequences of single binding sites (b.s.) for the appropriate miRNAs (ATXN3 b. s. for miR-9-2 and miR-181a-1) were cloned into the pmirGLO Dual-Luciferase miRNA Target Expression Vector (Promega). This vector, which was specially designed for miRNA-mRNA interaction studies, is based on Promega dual-luciferase technology, with firefly luciferase (luc2) as the primary reporter for monitoring mRNA regulation and Renilla luciferase (hRluc-neo) as a control reporter for normalization. Typically, in a reporter system, three types of constructs are prepared; a wild-type (WT) construct bearing an intact binding site(s) for the studied miRNA, a construct with mutations in its binding site(s) (MUT), and a perfect match (PM) construct (Figure 2A). Mutations in the sequence corresponding to the miRNA seed disrupt the native pairing within the binding region of the candidate miRNAs, which provides a negative control, whereas perfect complementarity with miRNA sequences induces target degradation and provides a positive control.

To validate the interactions between our candidate miRNAs, miR-9-2 and miR-181a-1, and the ATXN3 transcript, we transfected HEK293T cells with either reporter carrying potential miRNA binding sites. Four constructs were transfected into cells and tested in parallel. These constructs were co-transfected with appropriate miRNAcoding plasmid vectors (System Biosciences) because our experimental system required miRNA overexpression. The transient transfection of cells with reporter constructs was followed by measuring the reporter activity, which enabled the validation of predicted miRNA-mRNA interactions. Specifically, we obtained considerable repression of the luciferase expression after the transfection of reporter constructs for miR-181a-1, whereas no repression was measured after the transfection of reporters for miR-9-2 (Figure 2B).

The reduction in luciferase activity in the case of the WT construct carrying b.s. for miR-181a-1 was reproducible and statistically significant (suppression to 64\%). The PM construct repressed luciferase to a very low level, while the luciferase activity for the MUT construct showed efficient de-repression. In contrast, we did not observe any decrease in luciferase activity when the WT construct carrying b.s. for miR-9-2 was transfected to cells. As for the experimental procedures, enzymatic activities of firefly and Renilla luciferases were measured 


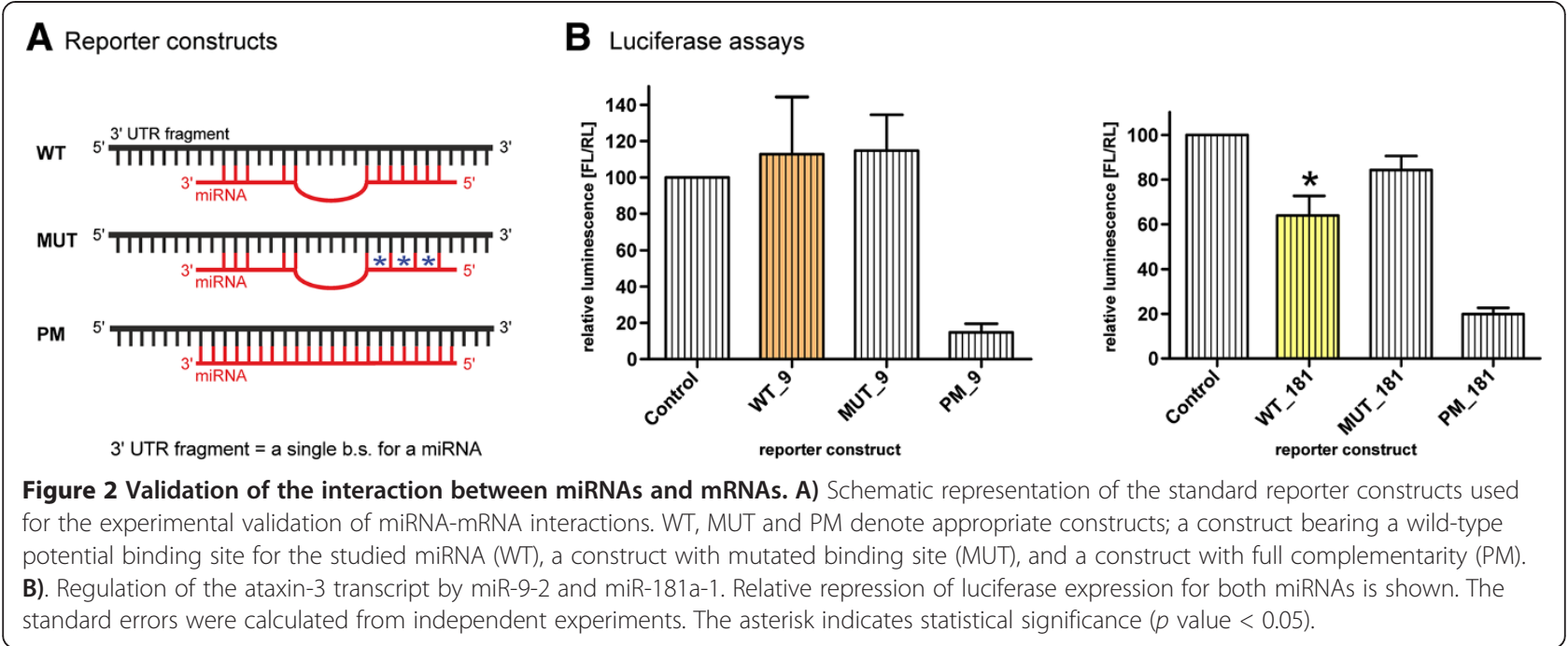

using a luminometer (Berthold Technologies) with the substrates and procedures from the Dual Luciferase Assay Kit (Promega). The values for firefly luciferase activity for every reporter construct were normalized to the corresponding values of Renilla luciferase activity to account for varying transfection efficiency. Relative expression values for all constructs were obtained by comparing their normalized luciferase activities with those of the control plasmid. Transfection was repeated 6 and 8 times for miR-9-2 and miR-181a-1, respectively.

Overall, using the luciferase reporter system, we have validated the interaction between miR-181a-1 and the 3 '-UTR of ATXN3 mRNA positively and the interaction between miR-9-2 and the 3'-UTR of ATXN3 mRNA negatively. This is only a preliminary validation of miRNAmRNA interactions with respect to SCA3 and needs to be elaborated farther, and it is an example of the utility of the reporter system in the search for miRNA targets. Nevertheless, this is the first evidence for the direct binding of miR-181a-1 to the 3'-UTR of the human ATXN3 gene.

\section{Conclusions}

Many studies have shown that miRNAs play a crucial role in the development and functional regulation of the nervous system, and the deregulation of miRNAs was postulated and proved in numerous neurological disorders of different etiology, including TREDs and even strictly polyQ diseases (reviewed in [47]). Modest but significant changes in miRNA expression were shown to influence severity and/or disease progression. Hence, the deregulation of miRNA expression is now being considered a hallmark of many diseases that has both prognostic and diagnostic value. All studies performed on the CAG repeat-dependent SCAs demonstrate changes in miRNA expression patterns in these disorders and, most importantly, suggest that miRNA pathways and polyQ- induced neurodegeneration are interdependent processes. There is accumulating evidence that miRNAs are important contributors to the pathogenicity associated with SCAs. Therefore, close attention should be paid to the therapeutic potential of miRNAs due to their regulatory activities. Both the miRNA-based therapy (e.g., the use of miRNA mimics, artificial miRNAs, miRNA inhibitors and sponges) as well as the implementation of miRNA-based biomarkers would be beneficial in the treatment of all SCAs. Moreover, in terms of therapy for these diseases particularly important is the fact that miRNAs may repress the levels of both toxicity factors, namely polyQ protein and mutant CAG repeat transcript. Of all the CAG repeat-dependent SCA types studied to date, however, most findings are related to SCA1 and, secondarily, to SCA3. The role of miRNAs in the pathogenesis of other CAG repeat-dependent SCAs and miRNAspecific functions remain largely unknown and require further investigation.

\section{Competing interests}

The authors declare that they have no competing interests.

\section{Authors' contributions}

EK designed and performed experiments, prepared figures and wrote the review paper. WJK supervised the research and contributed in the preparation of the paper. Both authors read and approved the final manuscript.

\section{Acknowledgements}

We would like to thank Urszula Czebreszuk for preparing the reporter constructs required for this study and for the preliminary validation of miR-9-2 and miR-181a-1 interaction with the ATXN3 transcript. This work was supported by funding from the Polish Ministry of Science and Higher Education (N N301 523038) and the National Science Centre (grant numbers 2011/03/B/NZ1/03259 and 2012/06/A/NZ1/00094).

Received: 11 March 2014 Accepted: 30 May 2014

Published: 7 August 2014 


\section{References}

1. Gatchel JR, Zoghbi HY: Diseases of unstable repeat expansion: mechanisms and common principles. Nat Rev Genet 2005, 6(10):743-755.

2. Orr HT: Cell biology of spinocerebellar ataxia. J Cell Biol 2012, 197(2):167-177.

3. Orr HT, Zoghbi HY: Trinucleotide repeat disorders. Annu Rev Neurosci 2007, 30:575-621.

4. Matilla-Duenas A, Ashizawa T, Brice A, Magri S, McFarland KN, Pandolfo M, Pulst SM, Riess O, Rubinsztein DC, Schmidt J, Schmidt T, Scoles DR, Stevanin G, Taroni F, Underwood BR, Sanchez I: Consensus Paper: Pathological Mechanisms Underlying Neurodegeneration in Spinocerebellar Ataxias. Cerebellum 2013, 13(2):269-302.

5. Matilla-Duenas A, Goold R, Giunti P: Clinical, genetic, molecular, and pathophysiological insights into spinocerebellar ataxia type 1. Cerebellum 2008, 7(2):106-114

6. Orr HT, Chung MY, Banfi S, Kwiatkowski TJ Jr, Servadio A, Beaudet AL, McCall AE, Duvick LA, Ranum LP, Zoghbi HY: Expansion of an unstable trinucleotide CAG repeat in spinocerebellar ataxia type 1. Nat Genet 1993, 4(3):221-226.

7. David G, Abbas N, Stevanin G, Durr A, Yvert G, Cancel G, Weber C, Imbert G, Saudou F, Antoniou E, Drabkin H, Gemmill R, Giunti P, Benomar A, Wood N, Ruberg M, Agid Y, Mandel JL, Brice A: Cloning of the SCA7 gene reveals a highly unstable CAG repeat expansion. Nat Genet 1997, 17(1):65-70

8. Imbert G, Saudou F, Yvert G, Devys D, Trottier Y, Garnier JM, Weber C, Mandel JL, Cancel G, Abbas N, Durr A, Didierjean O, Stevanin G, Agid Y, Brice A: Cloning of the gene for spinocerebellar ataxia 2 reveals a locus with high sensitivity to expanded CAG/glutamine repeats. Nat Genet 1996, 14(3):285-291.

9. Pulst SM, Nechiporuk A, Nechiporuk T, Gispert S, Chen XN, Lopes-Cendes I, Pearlman S, Starkman S, Orozco-Diaz G, Lunkes A, DeJong P, Rouleau GA, Auburger G, Korenberg JR, Figueroa C, Sahba S: Moderate expansion of a normally biallelic trinucleotide repeat in spinocerebellar ataxia type 2 . Nat Genet 1996, 14(3):269-276.

10. Kawaguchi Y, Okamoto T, Taniwaki M, Aizawa M, Inoue M, Katayama S, Kawakami H, Nakamura S, Nishimura M, Akiguchi, Kimura J, Narumiya S, Kakizuka A: CAG expansions in a novel gene for Machado-Joseph disease at chromosome 14q32.1. Nat Genet 1994, 8(3):221-228.

11. Zhuchenko O, Bailey J, Bonnen P, Ashizawa T, Stockton DW, Amos C, Dobyns WB, Subramony SH, Zoghbi HY, Lee CC: Autosomal dominant cerebellar ataxia (SCA6) associated with small polyglutamine expansions in the alpha $1 \mathrm{~A}$-voltage-dependent calcium channel. Nat Genet 1997, 15(1):62-69.

12. Nakamura K, Jeong SY, Uchihara T, Anno M, Nagashima K, Nagashima T, Ikeda S, Tsuji S, Kanazawa I: SCA17, a novel autosomal dominant cerebellar ataxia caused by an expanded polyglutamine in TATA-binding protein. Hum Mol Genet 2001, 10(14):1441-1448.

13. Mirkin SM: Expandable DNA, repeats and human disease. Nature 2007, 447(7147):932-940.

14. Lopez Castel A, Cleary JD, Pearson CE: Repeat instability as the basis for human diseases and as a potential target for therapy. Nat Rev Mol Cell Biol 2010, 11(3):165-170.

15. Davies SW, Turmaine M, Cozens BA, DiFiglia M, Sharp AH, Ross CA, Scherzinger $E$, Wanker EE, Mangiarini L, Bates GP: Formation of neuronal intranuclear inclusions underlies the neurological dysfunction in mice transgenic for the HD mutation. Cell 1997, 90(3):537-548.

16. Birman S: Neurodegeneration: RNA turns number one suspect in polyglutamine diseases. Curr Biol 2008, 18(15):R659-R661.

17. Li LB, Bonini NM: Roles of trinucleotide-repeat RNA in neurological disease and degeneration. Trends Neurosci 2010, 33(6):292-298.

18. Fiszer A, Krzyzosiak WJ: RNA toxicity in polyglutamine disorders: concepts, models, and progress of research. J Mol Med (Berl) 2013, 91(6):683-691.

19. Marti E, Estivill X: Small non-coding RNAs add complexity to the RNA pathogenic mechanisms in trinucleotide repeat expansion diseases. Front Mol Neurosci 2013, 6:45.

20. Griffiths-Jones S, Saini HK, Van Dongen S, Enright AJ: miRBase: tools for microRNA genomics. Nucleic Acids Res 2008, 36:D154-D158.

21. Chekulaeva M, Filipowicz W: Mechanisms of miRNA-mediated post-transcriptional regulation in animal cells. Curr Opin Cell Biol 2009, 21(3):452-460

22. Kim VN, Han J, Siomi MC: Biogenesis of small RNAs in animals. Nat Rev Mol Cell Biol 2009, 10(2):126-139.
23. Krol J, Loedige I, Filipowicz W: The widespread regulation of microRNA biogenesis, function and decay. Nat Rev Genet 2010, 11(9):597-610.

24. Winter J, Jung S, Keller S, Gregory RI, Diederichs S: Many roads to maturity: microRNA biogenesis pathways and their regulation. Nat Cell Biol 2009, 11(3):228-234.

25. Starega-Roslan J, Koscianska E, Kozlowski P, Krzyzosiak WJ: The role of the precursor structure in the biogenesis of microRNA. Cell Mol Life Sci 2011, 68(17):2859-2871.

26. Stroynowska-Czerwinska A, Fiszer A, Krzyzosiak WJ: The panorama of miRNA-mediated mechanisms in mammalian cells. Cell Mol Life Sci 2014, 71(12):2253-2270.

27. Burright EN, Clark HB, Servadio A, Matilla T, Feddersen RM, Yunis WS, Duvick LA, Zoghbi HY, Orr HT: SCA1 transgenic mice: a model for neurodegeneration caused by an expanded CAG trinucleotide repeat. Cell 1995, 82(6):937-948.

28. Cemal CK, Carroll CJ, Lawrence L, Lowrie MB, Ruddle P, Al-Mahdawi S, King RH, Pook MA, Huxley C, Chamberlain S: YAC transgenic mice carrying pathological alleles of the MJD1 locus exhibit a mild and slowly progressive cerebellar deficit. Hum Mol Genet 2002, 11(9):1075-1094.

29. Huynh DP, Figueroa K, Hoang N, Pulst SM: Nuclear localization or inclusion body formation of ataxin- 2 are not necessary for $\mathrm{SCA} 2$ pathogenesis in mouse or human. Nat Genet 2000, 26(1):44-50.

30. Xia H, Mao Q, Eliason SL, Harper SQ, Martins IH, Orr HT, Paulson HL, Yang L, Kotin RM, Davidson BL: RNAi suppresses polyglutamine-induced neurodegeneration in a model of spinocerebellar ataxia. Nat Med 2004, 10(8):816-820

31. Barbato C, Ruberti F, Cogoni C: Searching for MIND: microRNAs in neurodegenerative diseases. J Biomed Biotechnol 2009, 2009:871313.

32. Bushati N, Cohen SM: MicroRNAs in neurodegeneration. Curr Opin Neurobiol 2008, 18(3):292-296.

33. Hebert SS, De Strooper B: Alterations of the microRNA network cause neurodegenerative disease. Trends Neurosci 2009, 32(4):199-206.

34. Lau P, De Strooper B: Dysregulated microRNAs in neurodegenerative disorders. Semin Cell Dev Biol 2010, 21(7):768-773.

35. Martino S, Di Girolamo I, Orlacchio A, Datti A: MicroRNA implications across neurodevelopment and neuropathology. J Biomed Biotechnol 2009, 2009:654346.

36. Roshan R, Ghosh T, Scaria V, Pillai B: MicroRNAs: novel therapeutic targets in neurodegenerative diseases. Drug Discov Today 2009, 14(23-24):1123-1129.

37. Schaefer A, O'Carroll D, Tan CL, Hillman D, Sugimori M, Llinas R, Greengard P: Cerebellar neurodegeneration in the absence of microRNAs. J Exp Med 2007, 204(7):1553-1558.

38. Weinberg MS, Wood MJ: Short non-coding RNA biology and neurodegenerative disorders: novel disease targets and therapeutics. Hum Mol Genet 2009, 18(R1):R27-R39.

39. Eacker SM, Dawson TM, Dawson VL: The interplay of microRNA and neuronal activity in health and disease. Front Cell Neurosci 2013, 7:136.

40. Sun AX, Crabtree GR, Yoo AS: MicroRNAs: regulators of neuronal fate. Curr Opin Cell Biol 2013, 25(2):215-221.

41. Follert P, Cremer H, Beclin C: MicroRNAs in brain development and function: a matter of flexibility and stability. Front Mol Neurosci 2014, 7:5.

42. Delay C, Mandemakers W, Hebert SS: MicroRNAs in Alzheimer's disease. Neurobiol Dis 2012, 46(2):285-290.

43. Gascon E, Gao FB: Cause or Effect: Misregulation of microRNA Pathways in Neurodegeneration. Front Neurosci 2012, 6:48.

44. Esteller M: Non-coding RNAs in human disease. Nat Rev Genet 2011, 12(12):861-874

45. Gehrke S, Imai Y, Sokol N, Lu B: Pathogenic LRRK2 negatively regulates microRNA-mediated translational repression. Nature 2010, 466(7306):637-641.

46. Savas JN, Makusky A, Ottosen S, Baillat D, Then F, Krainc D, Shiekhattar R, Markey SP, Tanese N: Huntington's disease protein contributes to RNA-mediated gene silencing through association with Argonaute and P bodies. Proc Natl Acad Sci U S A 2008, 105(31):10820-10825.

47. Koscianska E, Kozlowska E, Jaworska E, Krzyzosiak W: MicroRNA Deregulation in Trinucleotide Repeat Expansion Disorders. In Applied RNAi: From Fundamental Research to Therapeutic Applications. Edited by Patrick A, Marc S. Weinberg: Caister Academic Press; 2014:227-246.

48. Lee Y, Samaco RC, Gatchel JR, Thaller C, Orr HT, Zoghbi HY: miR-19, miR-101 and miR-130 co-regulate ATXN1 levels to potentially modulate SCA1 pathogenesis. Nat Neurosci 2008, 11(10):1137-1139. 


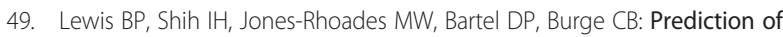
mammalian microRNA targets. Cell 2003, 115(7):787-798.

50. Persengiev S, Kondova I, Otting N, Koeppen AH, Bontrop RE: Genome-wide analysis of miRNA expression reveals a potential role for miR-144 in brain aging and spinocerebellar ataxia pathogenesis. Neurobiol Aging 2011, 32(12):2316 e17-27.

51. Kumar MS, Lu J, Mercer KL, Golub TR, Jacks T: Impaired microRNA processing enhances cellular transformation and tumorigenesis. Nat Genet 2007, 39(5):673-677.

52. Lu J, Getz G, Miska EA, Alvarez-Saavedra E, Lamb J, Peck D, Sweet-Cordero A, Ebert BL, Mak RH, Ferrando AA, Downing JR, Jacks T, Horvitz HR, Golub TR: MicroRNA expression profiles classify human cancers. Nature 2005, 435(7043):834-838.

53. Rodriguez-Lebron E, Liu G, Keiser M, Behlke MA, Davidson BL: Altered Purkinje cell miRNA expression and SCA1 pathogenesis. Neurobiol Dis 2013, 54:456-463.

54. Keiser MS, Geoghegan JC, Boudreau RL, Lennox KA, Davidson BL: RNAi or overexpression: alternative therapies for Spinocerebellar Ataxia Type 1. Neurobiol Dis 2013, 56:6-13.

55. Reinhardt A, Feuillette S, Cassar M, Callens C, Thomassin H, Birman S, Lecourtois M, Antoniewski C, Tricoire H: Lack of miRNA Misregulation at Early Pathological Stages in Drosophila Neurodegenerative Disease Models. Front Genet 2012, 3:226.

56. Bilen J, Liu N, Burnett BG, Pittman RN, Bonini NM: MicroRNA pathways modulate polyglutamine-induced neurodegeneration. Mol Cell 2006, 24(1):157-163.

57. Liu N, Landreh M, Cao K, Abe M, Hendriks GJ, Kennerdell JR, Zhu Y, Wang LS, Bonini NM: The microRNA miR-34 modulates ageing and neurodegeneration in Drosophila. Nature 2012, 482(7386):519-523.

58. Shi Y, Huang F, Tang B, Li J, Wang J, Shen L, Xia K, Jiang H: MicroRNA profiling in the serums of SCA3/MJD patients. Int J Neurosci 2013, 124(2):97-101.

59. Cheever A, Blackwell E, Ceman S: Fragile X protein family member FXR1P is regulated by microRNAs. RNA 2010, 16(8):1530-1539.

60. Gaughwin PM, Ciesla M, Lahiri N, Tabrizi SJ, Brundin P, Bjorkqvist M: Hsa-miR-34b is a plasma-stable microRNA that is elevated in pre-manifest Huntington's disease. Hum Mol Genet 2011, 20(11):2225-2237.

61. Pogue Al, Cui JG, Li YY, Zhao Y, Culicchia F, Lukiw WJ: Micro RNA-125b (miRNA-125b) function in astrogliosis and glial cell proliferation. Neurosci Lett 2010, 476(1):18-22.

62. Roshan R, Ghosh T, Gadgil M, Pillai B: Regulation of BACE1 by miR-29a/b in a cellular model of Spinocerebellar Ataxia 17. RNA Bio/ 2012, 9(6):891-899.

63. Shioya M, Obayashi S, Tabunoki H, Arima K, Saito Y, Ishida T, Satoh J: Aberrant microRNA expression in the brains of neurodegenerative diseases: miR-29a decreased in Alzheimer disease brains targets neurone navigator 3. Neuropathol Appl Neurobiol 2010, 36(4):320-330.

64. Sinha M, Ghose J, Das E, Bhattarcharyya NP: Altered microRNAs in STHdh (Q111)/Hdh(Q111) cells: miR-146a targets TBP. Biochem Biophys Res Commun 2010, 396(3):742-747.

65. McCann C, Holohan EE, Das S, Dervan A, Larkin A, Lee JA, Rodrigues V, Parker R, Ramaswami M: The Ataxin-2 protein is required for microRNA function and synapse-specific long-term olfactory habituation. Proc Nat Acad Sci U S A 2011, 108(36):E655-E662.

66. Fiszer A, Krzyzosiak WJ: Oligonucleotide-based strategies to combat polyglutamine diseases. Nucleic Acids Res 2014. Epub ahead of print.

67. Witkos TM, Koscianska E, Krzyzosiak WJ: Practical Aspects of microRNA Target Prediction. Curr Mol Med 2011, 11(2):93-109.

68. John B, Enright AJ, Aravin A, Tuschl T, Sander C, Marks DS: Human MicroRNA targets. PLoS Biol 2004, 2(11):e363.

69. Krek A, Grun D, Poy MN, Wolf R, Rosenberg L, Epstein EJ, MacMenamin P da Piedade I, Gunsalus KC, Stoffel M, Rajewsky N: Combinatorial microRNA target predictions. Nat Genet 2005, 37(5):495-500.

70. Lewis BP, Burge CB, Bartel DP: Conserved seed pairing, often flanked by adenosines, indicates that thousands of human genes are microRNA targets. Cell 2005, 120(1):15-20

71. Kruger J, Rehmsmeier M: RNAhybrid: microRNA target prediction easy, fast and flexible. Nucleic Acids Res 2006, 34:W451-W454.

72. Kertesz M, lovino N, Unnerstall U, Gaul U, Segal E: The role of site accessibility in microRNA target recognition. Nat Genet 2007, 39(10):1278-1284.
73. Rinck A, Preusse M, Laggerbauer B, Lickert H, Engelhardt S, Theis FJ: The human transcriptome is enriched for miRNA-binding sites located in cooperativity-permitting distance. RNA Bio/ 2013, 10(7):1125-1135.

74. Incarnato D, Neri F, Diamanti D, Oliviero S: MREdictor: a two-step dynamic interaction model that accounts for mRNA accessibility and Pumilio binding accurately predicts microRNA targets. Nucleic Acids Res 2013, 41(18):8421-8433.

75. Kuhn DE, Martin MM, Feldman DS, Terry AV Jr, Nuovo GJ, Elton TS: Experimental validation of miRNA targets. Methods 2008, 44(1):47-54.

76. Thomson DW, Bracken CP, Goodall GJ: Experimental strategies for microRNA target identification. Nucleic Acids Res 2011, 39(16):6845-6853.

77. Vasudevan S: Functional validation of microRNA-target RNA interactions. Methods 2012, 58(2):126-134

78. Esau C, Davis S, Murray SF, Yu XX, Pandey SK, Pear M, Watts L, Booten SL, Graham M, McKay R, Subramaniam A, Propp S, Lollo BA, Freier S, Bennett CF, Bhanot S, Monia BP: miR-122 regulation of lipid metabolism revealed by in vivo antisense targeting. Cell Metab 2006, 3(2):87-98.

79. Liu Z, Sall A, Yang D: MicroRNA: An emerging therapeutic target and intervention tool. Int J Mol Sci 2008, 9(6):978-999.

80. Wang X: Systematic identification of microRNA functions by combining target prediction and expression profiling. Nucleic Acids Res 2006, 34(5):1646-1652.

81. Ziegelbauer JM, Sullivan CS, Ganem D: Tandem array-based expression screens identify host mRNA targets of virus-encoded microRNAs. Nat Genet 2009, 41(1):130-134.

82. Konig J, Zarnack K, Luscombe NM, Ule J: Protein-RNA interactions: new genomic technologies and perspectives. Nat Rev Genet 2011, 13(2):77-83.

83. Miranda KC, Huynh T, Tay Y, Ang YS, Tam WL, Thomson AM, Lim B, Rigoutsos I: A pattern-based method for the identification of MicroRNA binding sites and their corresponding heteroduplexes. Cell 2006, 126(6):1203-1217.

84. Nicolas FE: Experimental validation of microRNA targets using a luciferase reporter system. Methods Mol Biol 2011, 732:139-152.

85. Hsu SD, Tseng YT, Shrestha S, Lin YL, Khaleel A, Chou CH, Chu CF, Huang HY, Lin CM, Ho SY, Jian TY, Lin FM, Chang TH, Weng SL, Liao KW, Liao IE, Liu CC, Huang HD: miRTarBase update 2014: an information resource for experimentally validated miRNA-target interactions. Nucleic Acids Res 2014, 42:D78-D85

86. Hsu SD, Lin FM, Wu WY, Liang C, Huang WC, Chan WL, Tsai WT, Chen GZ, Lee CJ, Chiu CM, Chien CH, Wu MC, Huang CY, Tsou AP, Huang HD: miRTarBase: a database curates experimentally validated microRNA-target interactions. Nucleic Acids Res 2011, 39:D163-D169.

87. Evers MM, Toonen LJ, Van Roon-Mom WM: Ataxin-3 Protein and RNA Toxicity in Spinocerebellar Ataxia Type 3: Current Insights and Emerging Therapeutic Strategies. Mol Neurobiol 2013, 49(3):1513-1531.

88. Ranum LP, Lundgren JK, Schut LJ, Ahrens MJ, Perlman S, Aita J, Bird TD, Gomez C, Orr HT: Spinocerebellar ataxia type 1 and Machado-Joseph disease: incidence of CAG expansions among adult-onset ataxia patients from 311 families with dominant, recessive, or sporadic ataxia. Am J Hum Genet 1995, 57(3):603-608.

89. Zoghbi HY, Orr HT: Pathogenic mechanisms of a polyglutamine-mediated neurodegenerative disease, spinocerebellar ataxia type 1. J Biol Chem 2009, 284(12):7425-7429.

90. Koscianska E, Baev V, Skreka K, Oikonomaki K, Rusinov V, Tabler M, Kalantidis K: Prediction and preliminary validation of oncogene regulation by miRNAs. BMC Mol Biol 2007, 8:79.

91. Kozlowska E, Krzyzosiak WJ, Koscianska E: Regulation of huntingtin gene expression by miRNA-137, $-214,-148 a$, and their respective isomiRs. Int J Mol Sci 2013, 14(8):16999-17016.

doi:10.1186/2053-8871-1-7

Cite this article as: Koscianska and Krzyzosiak: Current understanding of the role of microRNAs in spinocerebellar ataxias. Cerebellum \& Ataxias 2014 1:7. 